\title{
To revascularize or not before transcatheter aortic valve implantation?
}

\author{
Sergio Perez ${ }^{1}$, Torin P. Thielhelm ${ }^{2}$, Mauricio G. Cohen ${ }^{1}$ \\ ${ }^{1}$ Cardiovascular Division, University of Miami Miller School of Medicine, Miami, FL, USA; ${ }^{2}$ University of Miami Miller School of Medicine, \\ Miami, FL, USA \\ Contributions: (I) Conception and design: All authors; (II) Administrative support: All authors; (III) Provision of study materials or patients: MG \\ Cohen; (IV) Collection and assembly of data: All authors; (V) Data analysis and interpretation: None; (VI) Manuscript writing: All authors; \\ (VII) Final approval of manuscript: All authors. \\ Correspondence to: Mauricio G. Cohen, MD. University of Miami Hospital \& Clinics, UHealth Tower, 1400 NW 12th Avenue, Suite 864, Miami, FL \\ 33136, USA. Email: mgcohen@med.miami.edu.
}

\begin{abstract}
Concomitant coronary artery disease (CAD) and aortic stenosis occur in approximately 60-75\% of patients referred for surgical or transcatheter aortic valve replacement (TAVR). Current guidelines support simultaneous surgical aortic valve replacement and bypass surgery with a class IIa recommendation, based on observational, non-randomized data. With the inception of TAVR, this strategy has been challenged, as observational studies have not shown significant outcome differences in patients with and without CAD treated with TAVR. Performing percutaneous coronary intervention (PCI) in patients with aortic stenosis is safe, but the indication and timing remain controversial. Complete revascularization before TAVR with low residual Syntax score $(<8)$ may be considered in selected cases with extensive, proximal, and severe CAD to improve outcomes. PCI before TAVR may require less contrast and reduce the risk of acute kidney injury, but uninterrupted dual antiplatelet therapy may increase the risk of bleeding during TAVR. Combined PCI and TAVR can be considered for unstable patients with simple lesions or ostial lesions, with risk of coronary occlusion after deployment of the transcatheter heart valve. PCI after TAVR may be considered in patients who remain symptomatic with significant residual ischemia despite optimal medical therapy. In the near future, it is expected that randomized clinical trials will further clarify the indications and role of revascularization in patients undergoing TAVR. In this article we provide an extensive review on the management of CAD in TAVR candidates, including additional considerations on technical aspects, device selection, and adjunctive pharmacological therapies.
\end{abstract}

Keywords: Transcatheter aortic valve replacement (TAVR); coronary artery disease (CAD); aortic stenosis (AS); revascularization; percutaneous coronary intervention (PCI)

Submitted Jul 29, 2018. Accepted for publication Sep 18, 2018.

doi: $10.21037 /$ jtd.2018.09.85

View this article at: http://dx.doi.org/10.21037/jtd.2018.09.85

\section{Prevalence of coronary artery disease in patients with aortic valvular disease}

Atherosclerosis and aortic stenosis (AS) have similar risk factors; consequently, concomitant coronary artery disease (CAD) is often found in patients with symptomatic severe AS. The presence of CAD has been reported in up to $60 \%$ of patients referred for evaluation for aortic valve replacement (AVR) $(1,2)$. Traditionally, the management strategy for these patients has been surgical AVR (SAVR) and coronary artery bypass grafting (CABG) in a single procedure. Despite the lack of randomized clinical data comparing SAVR with and without concomitant CABG, current guidelines for management of patients with valvular heart disease give a class IIa recommendation for revascularization of major coronary arteries with 
$>70 \%$ luminal stenosis or left main artery with $>50 \%$ luminal stenosis at the time of valve replacement. These recommendations are based on observational data that suggests improved outcomes in patients who underwent left internal mammary artery (LIMA) grafting to the left anterior descending artery (LAD) at the time of $\operatorname{AVR}(3,4)$.

Since its initial approval for clinical use in the United States by the Food and Drug Administration (FDA) in November 2011 and the Centers for Medicare and Medicaid Services (CMS) in May 2012, transcatheter AVR (TAVR) has emerged as an established treatment for patients with severe AS at prohibitive, high, or intermediate surgical risk. Approximately $40-75 \%$ of patients referred for TAVR have significant coronary artery disease, $12-51 \%$ of patients have a history of prior myocardial infarction, and $14-48 \%$ of patients have had some form of revascularization, surgical or percutaneous (5). Despite the significant prevalence of CAD in this patient population, $\mathrm{MI}$ as a complication of TAVR is an uncommon event occurring in $<1-4 \%$ of cases (6). However, the potential benefits of coronary revascularization prior to TAVR, including the reduction and prevention of myocardial ischemia during periods of transient hypotension throughout the procedure, among others, have motivated research aiming to determine if there is a real clinical benefit from revascularization. Currently, there is no standard treatment strategy for these patients. The randomized clinical trials that served as the foundation for the initial approval of TAVR for clinical use, PARTNER I and US CoreValve High Risk Study, excluded patients with non-revascularized CAD. Thus, the performance of PCI in patients with extensive and severe CAD before TAVR is not an uncommon practice.

The management of concomitant CAD in patients evaluated for TAVR can be complex and challenging. The elevated afterload imposed by AS and ventricular hypertrophy results in subendocardial ischemia due to vasoconstriction and mismatch between myocardial oxygen supply and demand, even in the absence of epicardial CAD. The high outflow resistance state in AS impairs left ventricular emptying and increases the end diastolic pressure with a consequent decrease in coronary flow (7). For these reasons, the presenting symptoms and results of non-invasive myocardial perfusion studies and coronary flow measurements (e.g., fractional flow reserve) are challenging to interpret in patients with severe AS (8).

\section{Impact of concomitant CAD on outcomes}

Earlier studies evaluating the impact of concomitant CAD in patients undergoing TAVR reported dissimilar results. Dewey et al. assessed the impact of CAD in 171 patients with history of CABG or PCI undergoing TAVR and showed a higher 30-day (13.1\% vs. $1.2 \%, \mathrm{P}=0.002)$ and 1 -year mortality (35.7\% vs. $18.4 \%, \mathrm{P}=0.01$ ) in patients with $\mathrm{CAD}(9)$. On the other hand, Masson et al. found no significant differences in 30-day or 1-year mortality, nor in left ventricular systolic function recovery, in 136 patients with and without CAD when stratified by CAD severity based on Duke Myocardial Jeopardy Score (DMJS) (10). Similar results were reported by a Swiss group that performed TAVR and PCI either staged or as a single procedure in 59 of 256 patients with CAD. No significant difference in 30-day mortality was found $(10.2 \%$ vs. $5.6 \%, \mathrm{P}=0.24)$ (11). The multicenter German registry with 1,382 patients reported a higher in-hospital mortality in patients with CAD (10\% vs. $5.5 \%$, OR 1.90, 95\% CI: 1.23-2.93), but this mortality difference was no longer significant after adjusted analysis (adjusted OR 1.41, 95\% CI: 0.85-2.33) (12). Other observational studies also failed to demonstrate differences in outcomes in patients with and without CAD (13). A recent meta-analysis that included over 2,472 patients in seven non-randomized observational studies reported similar all-cause mortality rates in patients with and without CAD undergoing TAVR (2).

\section{Impact of revascularization before TAVR}

As compared to earlier studies that aimed to determine if the presence of concomitant CAD would affect clinical outcomes of patients undergoing TAVR, subsequent studies intended to elucidate if percutaneous revascularization could attenuate the potential adverse effects of CAD in long-term outcomes.

Historically, PCI was not commonly performed in patients with severe AS because it was believed to be associated with a higher risk of procedural complications; however, Goel et al. demonstrated that PCI can be safely performed in patients with severe AS without significant increase in mortality compared to patients without aortic valvular disease (14). Abdel-Wahab et al. performed PCI within 10 days prior to TAVR in 55 patients and found no significant differences in 30-day or 6-month mortality when compared to 70 patients who underwent TAVR without preceding PCI (12).

The significance of the severity and extent of CAD in patients undergoing TAVR was evaluated by Paradis et al. A total of 337 patients were stratified in 4 groups according to their SYNTAX score (SS). Neither the presence nor the 


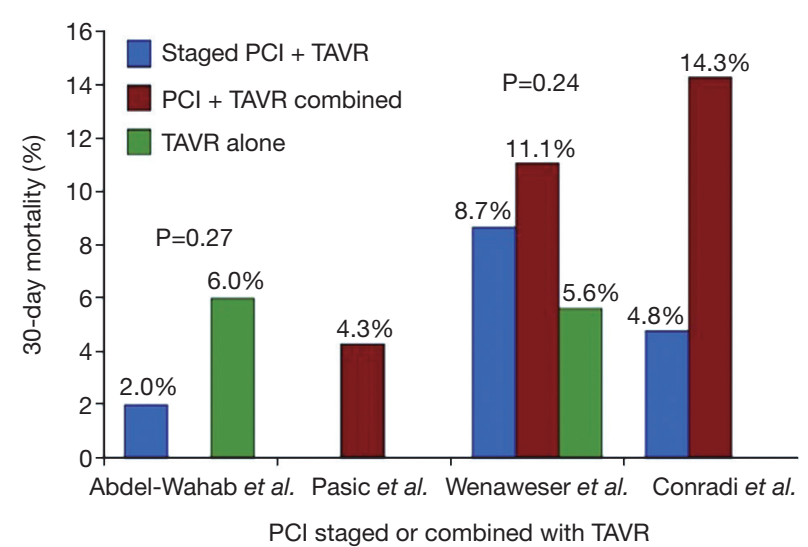

Figure 1 Mortality comparison between staged PCI and TAVR and PCI combined with TAVR in a single procedure. From Goel et al. (5). PCI, percutaneous coronary intervention; TAVR, transcatheter valve replacement.

severity of CAD had an impact on the rate of a combined primary end-point of all-cause mortality, cardiovascular mortality, and myocardial infarction at 30 days and 1 year after TAVR. There were no significant differences between patients with less complete revascularization (residual SS >8) and those with more complete revascularization (residual SS <8) (15). In contrast, another study of similar design by Witberg et al. showed that it is the severity of CAD and not the mere presence of the disease alone that is associated with a worse prognosis. Therefore, complete revascularization before TAVR could attenuate its effects (16). The same group recently published a multicenter study with 1,270 patients classified according to severity of $\mathrm{CAD}$ [no $\mathrm{CAD}$, non-severe $\mathrm{CAD}$ ( $\mathrm{SS}<22$ ), severe $\mathrm{CAD}(\mathrm{SS}>22)$ ] and revascularization completeness (residual $\mathrm{SS}<8$ vs. residual $\mathrm{SS}>8$ ). A higher all-cause mortality was seen in the severe CAD (SS >22) and incomplete revascularization (residual $\mathrm{SS}>8$ ) groups over a median follow up of 1.9 years but not in the non-severe $\mathrm{CAD}$ $(\mathrm{SS}<22$ ) or less incomplete revascularization (residual SS $<8$ ) groups when compared to patients with no CAD. The effect of severe $\mathrm{CAD}$ and incomplete revascularization on patient outcomes was independent of vascular access, baseline renal function, Society of Thoracic Surgeons (STS) score, age or left ventricular systolic function (17).

In summary, data have shown contradictory results; however, it has been clearly demonstrated that, if needed, PCI can be safely performed before TAVR in patients with severe CAD without a significant increase in the risk of complications and may even improve the overall prognosis in selected cases.

\section{Left main disease}

Historically, patients with left main coronary artery (LM) disease are considered high risk and are thought to be more vulnerable to hemodynamic deterioration during TAVR. Furthermore, the risk of LM occlusion by the prosthesis or the native leaflets after valve implantation is always a concern due to the anatomic proximity of the aortic valve annulus to the ostium of the artery.

The TAVR-LM registry evaluated 204 patients with coexisting aortic valve disease and LM disease undergoing TAVR plus PCI. Patients were classified based on PCI strategy (planned or not planned) and timing with TAVR (before, during, or after). Overall, 1-year mortality was similar between patients who underwent TAVR plus LM PCI and a matched cohort of patients undergoing TAVR without LM revascularization, except in patients who underwent unplanned emergency LM PCI because of TAVR-related complications (e.g., stent-related coronary obstruction or LM dissection); this group had a higher 30-day and 1-year mortality, as well as higher incidence of cardiogenic shock, need for cardiopulmonary resuscitation, and acute renal failure. Patients undergoing unplanned LM PCI often had higher residual mean and peak gradients, lower LM height, and were more likely to have multivessel CAD and need valve-in-valve procedures (18). Based on these data, it is reasonable to conclude that planned LM PCI before or during TAVR does not result in incremental risk compared with TAVR alone; therefore, a careful and comprehensive evaluation is important in order to anticipate possible complications. In selected cases, operators should consider protecting the LM ostium with a wire and an undeployed coronary stent placed in the distal LM or one of its branches, should a complication occur during the valve implant.

\section{What is the best timing of revascularization in patients undergoing TAVR?}

Once PCI has been deemed necessary because CAD severity warrants an intervention, what is the best timing for revascularization? Before, during or after TAVR? Overall, data from different observational studies have shown that both strategies of performing PCI either before or after TAVR in a combined procedure or staged into different procedures are feasible and yield similar results $(11,12,19,20)$ (Figure 1). However, it is important to highlight possible advantages and disadvantages of each strategy. Staged PCI 

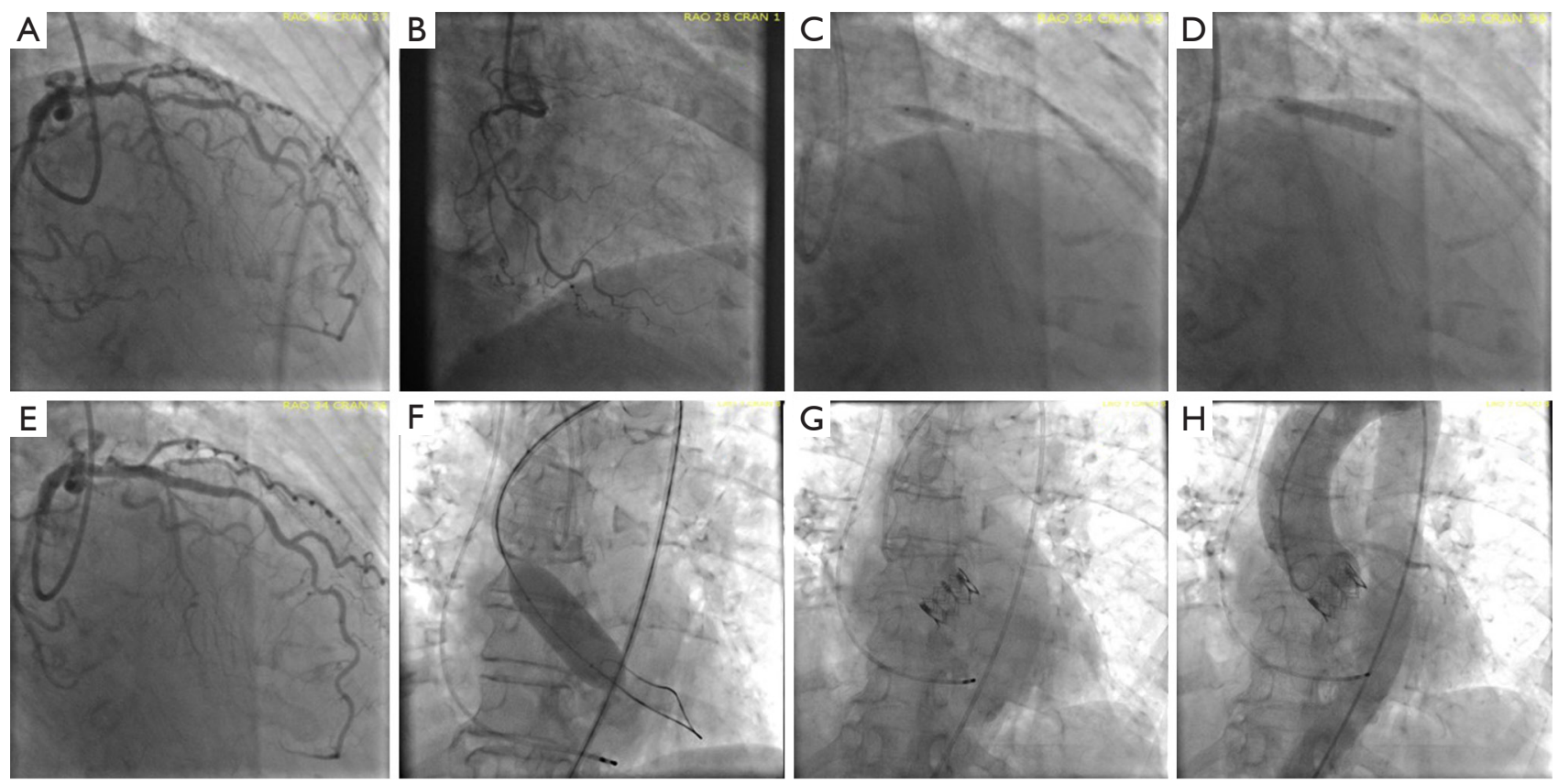

Figure 2 PCI before TAVR. This is an 88-year-old woman with severe AS who presents with worsening angina and shortness of breath. (A,B) Coronary angiography revealed severe stenosis of the proximal left anterior descending artery and small, diffusely diseased non-dominant right coronary artery; (C) predilation using a $3.0 \mathrm{~mm} \times 15 \mathrm{~mm}$ balloon; (D) deployment of drug-eluting stent in the proximal left anterior descending artery (resolute $3.5 \mathrm{~mm} \times 22 \mathrm{~mm}$ ); (E) final result after percutaneous coronary intervention. Two months later she underwent TAVR for treatment of AS; (F) balloon aortic valve dilation; (G) deployment of $23 \mathrm{~mm}$ Edwards XT valve; (H) final result with non-selective injection showing patency of the left main. The patient was discharged home the following day. PCI, percutaneous coronary intervention; TAVR, transcatheter valve replacement; AS, aortic stenosis.

before TAVR implies a lower contrast volume given at a procedural stage, reducing the risk of contrast-induced nephropathy. It can also minimize the risk of myocardial ischemia during rapid ventricular pacing for balloon valve dilation or valve deployment (Figure 2). On the other hand, the need for uninterrupted dual antiplatelet therapy after PCI could increase the risk of bleeding complications during TAVR, particularly if an alternative vascular access to the femoral artery is used (i.e., direct aortic or transapical). In addition, the risk of procedural complications during PCI can be higher in the presence of non-treated severe AS.

Combined PCI and TAVR may be an adequate strategy for unstable patients with very elevated aortic gradients at high risk to undergo PCI alone. It is also preferred in cases of simple lesions or in patients with ostial lesions with risk of coronary occlusion. A combined procedure only requires vascular access in a single episode of care, minimizing the risk of vascular complications and avoiding the need for uninterrupted dual antiplatelet therapy at the time of the procedure; however, a combined procedure increases procedural time, radiation exposure, and contrast load (Table 1).

An analysis of 22,344 patients using the Nationwide Inpatient Sample reported a higher in-hospital mortality and an increase in vascular complications in patients who received PCI during the same hospitalization in which they underwent TAVR when compared with patients that underwent only TAVR during their hospitalization (21). A recent meta-analysis that included a total of 3,858 patients from 9 studies found an increase in vascular complications (OR 1.86; 95\% CI, 1.33-2.60) and a higher 30-day all-cause mortality (OR 1.41; 95\% CI, 1.08-1.87) in patients that underwent PCI before or combined with TAVR when compared with patients that underwent TAVR without coronary revascularization. However, the authors found no significant differences in the rates of myocardial infarction, acute renal failure, stroke, or cardiovascular mortality at 30-day and 1-year follow-up (Figure 3). There were no differences in outcomes when PCI was performed before or during TAVR (22).

PCI may be needed after TAVR in cases with significant 
Table 1 Advantages and disadvantages of PCI before, during, and after TAVR

\begin{tabular}{|c|c|c|}
\hline Timing of $\mathrm{PCl}$ & Pro & Con \\
\hline \multirow[t]{2}{*}{$\mathrm{PCl}$ before TAVR } & $\begin{array}{l}\text { Coronary access easier without the presence of valvular } \\
\text { prosthesis }\end{array}$ & DAPT after PCI may increase bleeding after TAVR \\
\hline & Minimizes contrast loading in the same procedure & \\
\hline $\mathrm{PCl}$ at the time of TAVR & $\begin{array}{l}\text { Lower risk of hemodynamic compromise and ischemia } \\
\text { during TAVR }\end{array}$ & \\
\hline $\mathrm{PCl}$ after TAVR & $\begin{array}{l}\text { Treating aortic stenosis may improve myocardial } \\
\text { perfusion, removing the need for } \mathrm{PCl}\end{array}$ & $\begin{array}{l}\text { Problems with access; frame of valve can interfere } \\
\text { with coronary access }\end{array}$ \\
\hline
\end{tabular}

$\mathrm{PCl}$, percutaneous coronary intervention; DAPT, dual antiplatelet therapy; TAVR, transcatheter valve replacement.

\section{0-day, 1-year and cardiovascular mortality}

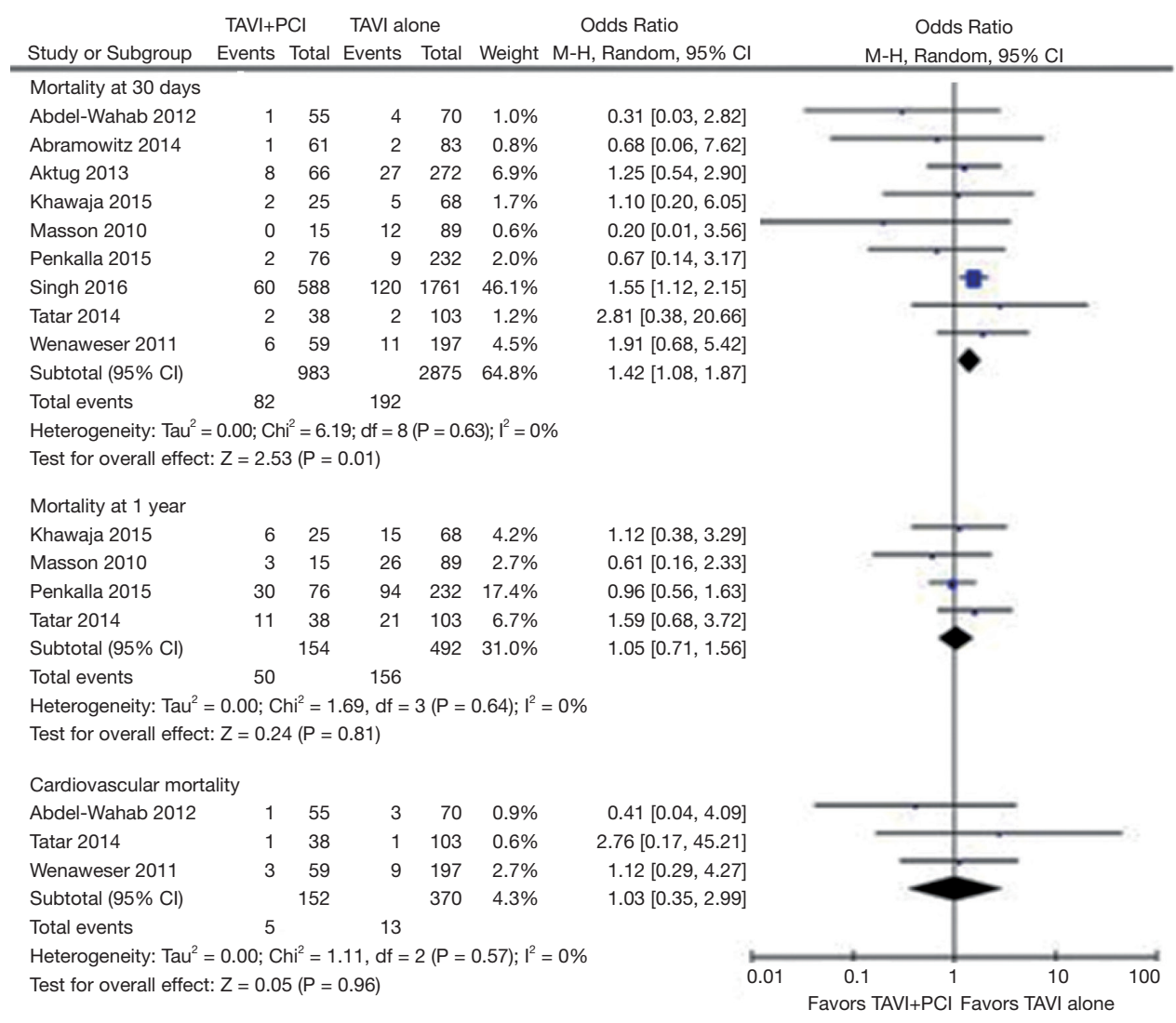

Figure 3 Mortality at 30-day and 1-year in patients with and without PCI before TAVR. From Kotronias et al. (22). PCI, percutaneous coronary intervention; TAVR, trans catheter valve replacement. 

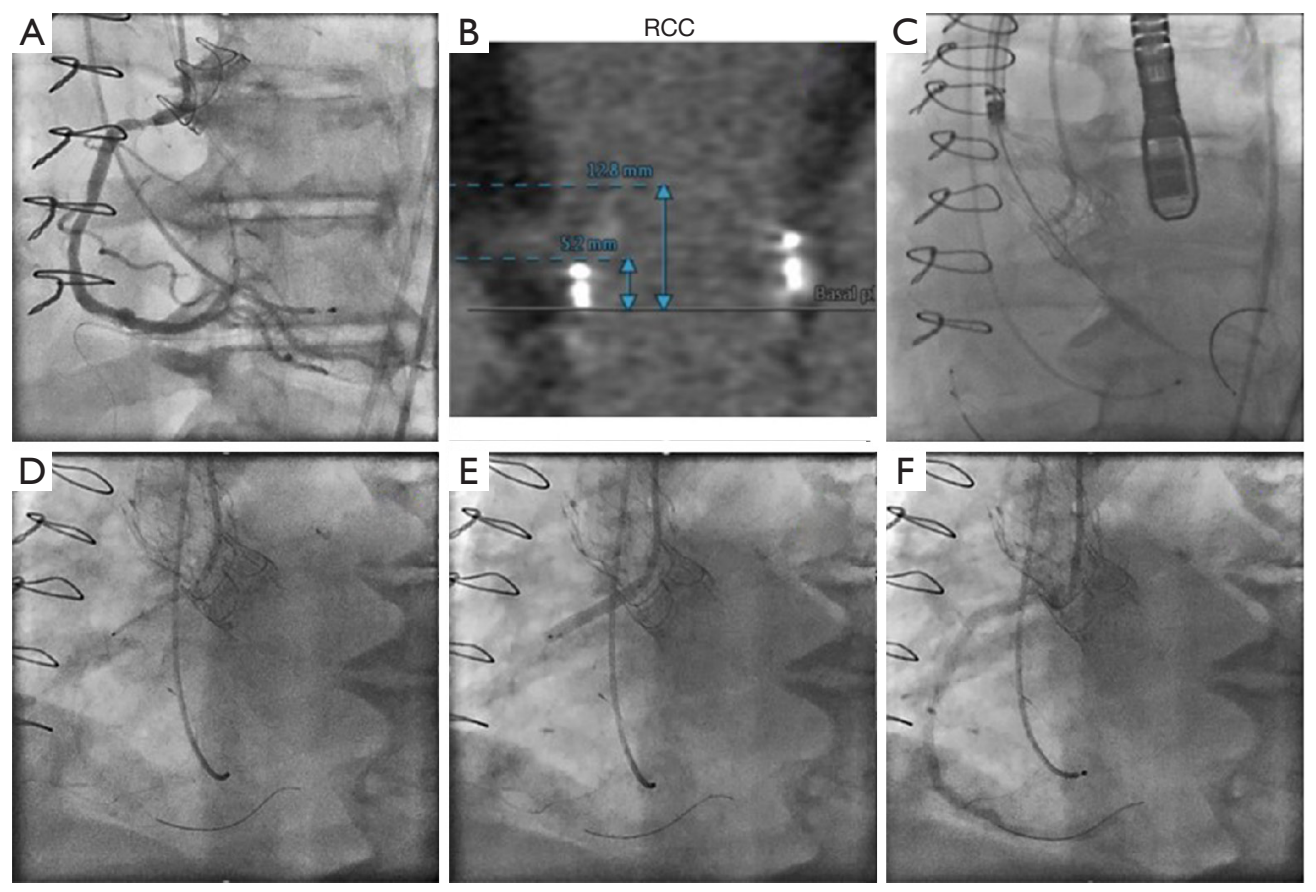

Figure 4 PCI during TAVR. Inoperable patient with severe stenosis of aortic valve bioprosthesis and disease of the proximal right coronary artery. TAVR and PCI were performed in the same procedure. (A,B) Valve-in-valve procedure, a small Valsalva sinus and a low origin of the right coronary artery in relation to the valve plane are risk factors for coronary occlusion during TAVR; (C) implant of 23-mm CoreValve Evolute R aortic prosthesis while performing coronary protection by positioning a 0.014 " guidewire and a stent (resolute $3.5 \mathrm{~mm} \times 28 \mathrm{~mm}$ ) without releasing into the coronary artery; (D) positioning of the stent in the coronary ostium after valvular implant; (E) stent deployment; (F) final result. PCI, percutaneous coronary intervention; TAVR, transcatheter valve replacement.

residual myocardial ischemia. If there is a possibility that a patient may require PCI after TAVR, the selection of a transcatheter valve prosthesis that does not block access to the coronary ostium (i.e., Lotus or Sapien) is an important consideration in procedural planning (23).

\section{Technical considerations}

The limitations of visual estimation of lesion severity from angiograms of calcified and tortuous coronary arteries commonly seen in patients with severe AS should be noted. The decision to perform PCI should be balanced against the potential increased risk of the procedure. If adding PCI implies a significantly increased risk of procedural complications, for instance as in patients with severely depressed left ventricular systolic function or patients with an elevated STS score, the decision to perform PCI should be revised. Another aspect to consider is the choice of drug-eluting or bare-metal stents based on the patient's bleeding risk. In the current era the vast majority of patients can be safely treated with newer generation drug-eluting stents, because the risk of stent thrombosis is minimal even after early discontinuation of dual antiplatelet therapy (24). Other important technical aspects include the localization of the lesion, the morphologic complexity of the atherosclerotic plaque, and the accessibility to the coronary arteries. The choice of valve prosthesis, the width of the sinuses of Valsalva, and the relationship of the valve annulus to the ostium of the coronary arteries should be carefully evaluated with computed tomography. Large coronary sinuses are preferred when choosing a self-expandable CoreValve prosthesis to avoid coronary occlusion. In patients with a low coronary takeoff $(<10 \mathrm{~mm}$ from the valve annulus) or at high risk of coronary occlusion, it is recommended to protect the coronary artery by advancing a 0.014 " coronary wire and a coronary stent that can be positioned in the left main or one of its branches, and deployed in the coronary ostium, in the event of coronary obstruction after valve deployment (Figure 4). In patients with previous ostial stents, valve deployment should be done 

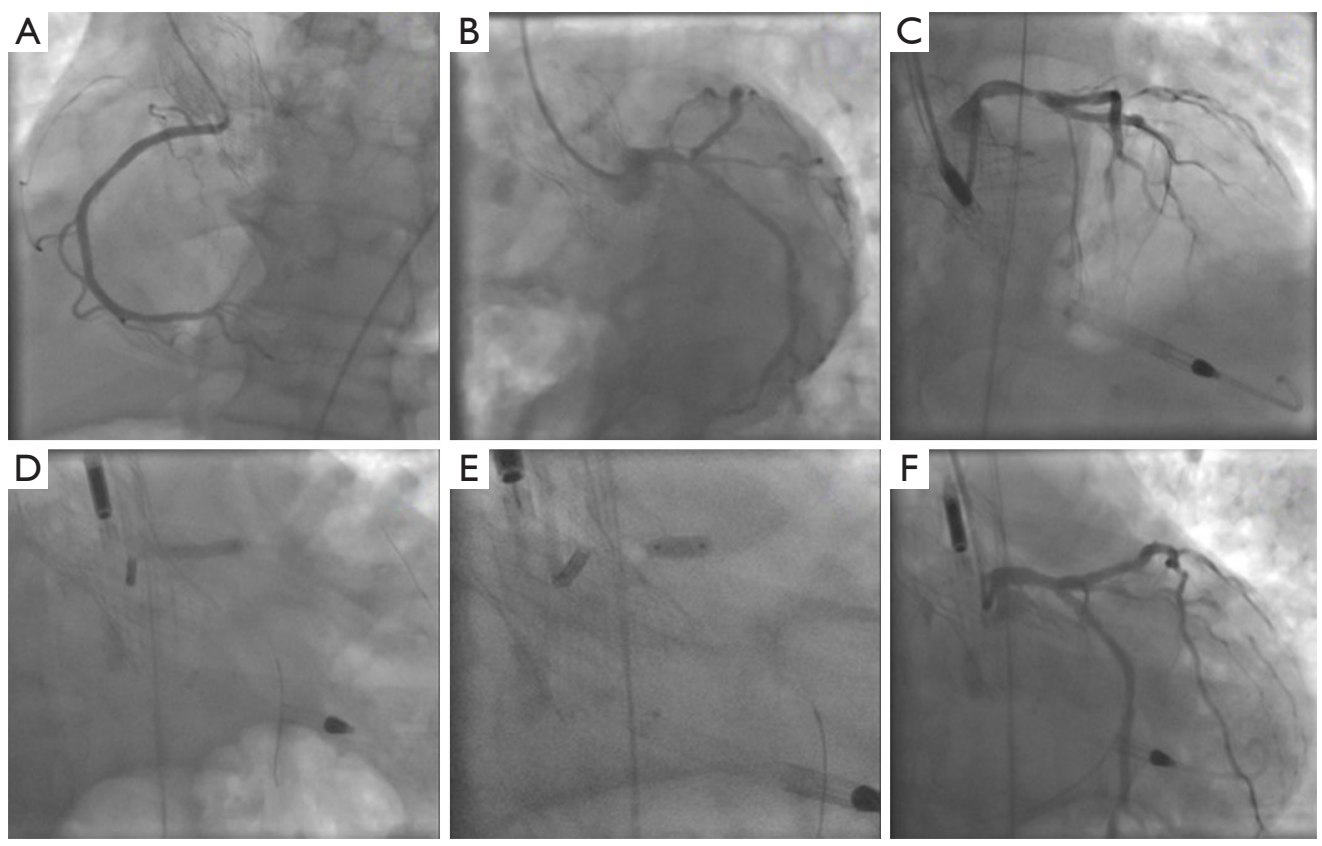

Figure 5 PCI after TAVR. This is an 83-year-old patient who had TAVR with a 31-mm CoreValve 2 years ago that now presents with congestive heart failure symptoms and non-ST elevation myocardial infarction. The ejection fraction was 30\%. (A,B) Coronary angiography revealed significant stenosis in the distal left main involving the bifurcation and origin of the left anterior descending artery and left circumflex artery. Cannulation of coronary ostia was performed in a coaxial manner through the diamonds of the valve frame using JR4 and JL4 catheters for the right and left coronary artery respectively; (C) an Impella CP catheter was advanced into the left ventricle for mechanical circulatory support during high-risk PCI in the setting of significant left ventricular dysfunction. Using an EBU 3.0 guidecatheter, Prowater coronary wires were advanced into the left anterior descending artery and the left circumflex; (D) balloon angioplasty and a provisional side-branch stenting strategy, using a resolute $3.5 \mathrm{~mm} \times 15 \mathrm{~mm}$ placed in the left main slightly extending into the proximal left anterior descending artery; (E) stent post dilation using a $3.5 \mathrm{~mm} \times 8 \mathrm{~mm}$ non-compliant balloon; (F) final result showed patency of both branches without need to stent the left circumflex ostium. PCI, percutaneous coronary intervention; TAVR, transcatheter valve replacement.

carefully to avoid crushing or deforming the stent struts (25).

Coronary artery cannulation and PCI can be challenging after TAVR. Each transcatheter aortic valve prosthesis requires specific technical considerations, and careful computed tomography analysis is important for procedure planning. With the self-expanding CoreValve with a design that extends above the coronary ostia, coronary engagement occurs through the prosthetic valve cells (Figure 5). Guiding catheter manipulation and coronary engagement with extra-backup curves should be done carefully through the CoreValve cells, as cases of catheter entrapment by the prosthesis struts have been described (26). Difficult selective coronary cannulation after high valve implants can occur in patients with low coronary ostia, with self- and balloonexpandable transcatheter valves. Coronary access can be impaired by a redundant long native leaflet or a misaligned commissural frame post in front of the coronary ostium.
On occasion, the Sapien S3 valve can extend proximally to the sino-tubular junction making coronary access very challenging (23).

\section{Conclusions}

In summary, the management of significant CAD in patients with severe AS undergoing TAVR is challenging and still has unsolved questions. The need for randomized clinical trials aiming to elucidate how to best treat these patients is evident in view of contradicting results yielded from observational studies. The ACTIVATION trial (The percutaneous coronary intervention prior to transcatheter aortic valve implantation), a European multi-center clinical trial that enrolled and randomized 310 patients with CAD to be treated with or without PCI before TAVR, is highly anticipated (27). The Danish NOTION-3 trial 


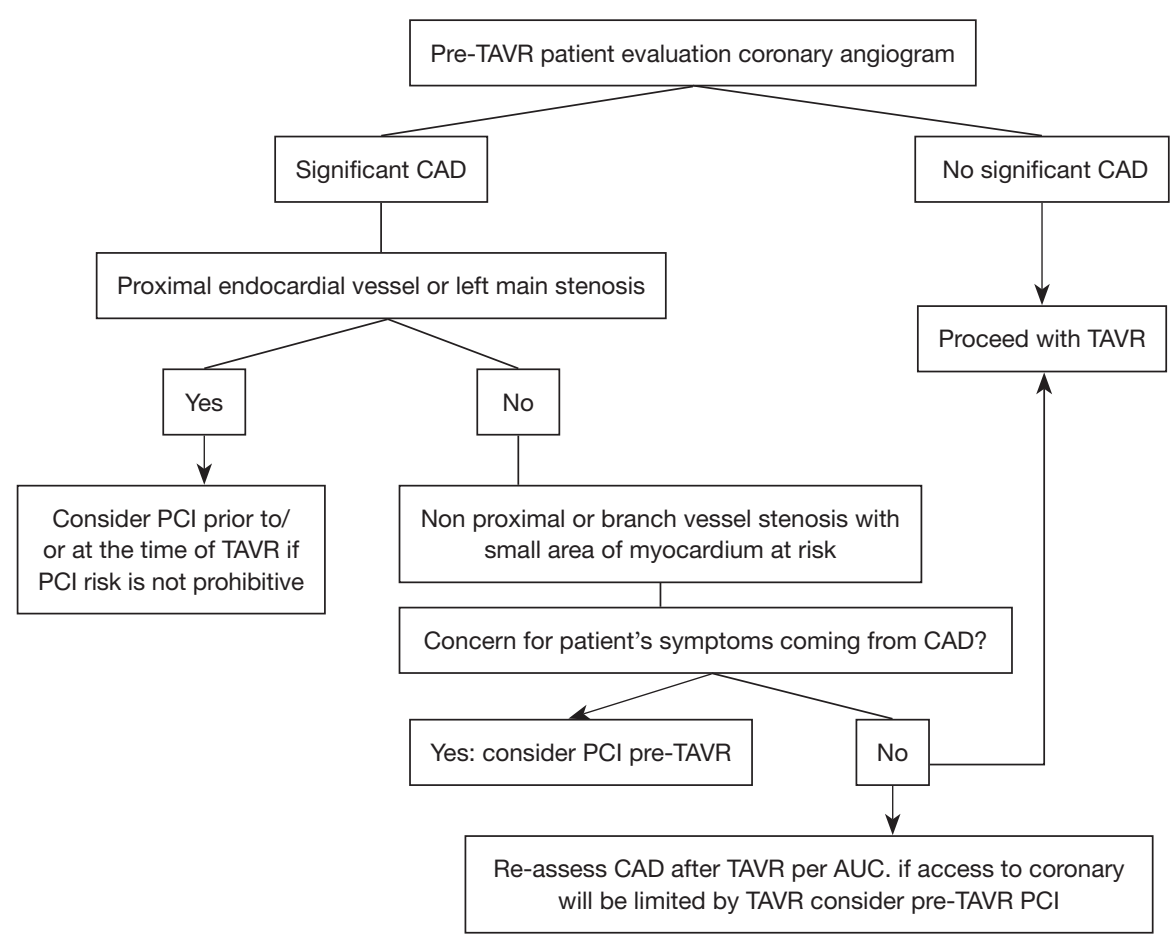

Figure 6 Treatment algorithm for patient candidates for TAVR with significant CAD. From Ramee et al. (28). PCI, percutaneous coronary intervention; CAD, coronary artery disease; AUC, appropriateness use criteria; TAVR, transcatheter valve replacement.

(NCT03058627) will compare complete revascularization guided by fractional flow reserve versus no revascularization in patients undergoing TAVR. The results of these studies may allow more informed treatment decisions in the future.

For now, the management of these patients must be individualized based on risk profile, clinical condition, particular anatomy, and operator experience. Most operators are in agreement that coronary revascularization should be considered before TAVR when CAD involves proximal segments of major epicardial coronary arteries. The Interventional Section Leadership Council of the American College of Cardiology proposed a treatment algorithm (Figure 6) in which the decision to perform PCI is reserved for patients with proximal epicardial coronary stenosis $>70 \%$ or left main stenosis $>50 \%$, provided the benefits from PCI outweigh the risk of the procedure. The algorithm recommends deferring revascularization of disease involving non-proximal segments or branch vessels with small areas of myocardium at risk unless the patient's symptoms are coming primarily from CAD or if future access to the coronary will be limited by TAVR (28). In general, it is recommended to perform PCI before TAVR in patients with significant renal dysfunction in whom the contrast load must be minimized and in those patients in whom there is no concern about prolonged dual antiplatelet therapy. PCI is recommended concomitantly with TAVR in patients with a high risk of coronary obstruction (e.g., ostial lesions, low LM height, or a valve-in-valve implant) or in patients in whom it is desirable to minimize dual antiplatelet therapy due to bleeding risk.

\section{Acknowledgements}

None.

\section{Footnote}

Conflicts of Interest: The authors have no conflicts of interest to declare.

\section{References}

1. Kvidal P, Bergstrom R, Horte LG, et al. Observed and relative survival after aortic valve replacement. J Am Coll Cardiol 2000;35:747-56.

2. D'Ascenzo F, Conrotto F, Giordana F, et al. Mid-term 
prognostic value of coronary artery disease in patients undergoing transcatheter aortic valve implantation: a meta-analysis of adjusted observational results. Int J Cardiol 2013;168:2528-32.

3. Nishimura RA, Otto CM, Bonow RO, et al. 2014 AHA/ ACC guideline for the management of patients with valvular heart disease: a report of the American College of Cardiology/American Heart Association Task Force on Practice Guidelines. J Thorac Cardiovasc Surg 2014;148:e1-132. Erratum in: J Thorac Cardiovasc Surg 2014;64:1763. Dosage error in article text.

4. Thalji NM, Suri RM, Daly RC, et al. The prognostic impact of concomitant coronary artery bypass grafting during aortic valve surgery: implications for revascularization in the transcatheter era. J Thorac Cardiovasc Surg 2015;149:451-60.

5. Goel SS, Ige M, Tuzcu EM, et al. Severe aortic stenosis and coronary artery disease--implications for management in the transcatheter aortic valve replacement era: a comprehensive review. J Am Coll Cardiol 2013;62:1-10.

6. Smith CR, Leon MB, Mack MJ, et al. Transcatheter versus surgical aortic-valve replacement in high-risk patients. $\mathrm{N}$ Engl J Med 2011;364:2187-98.

7. Rajappan K, Rimoldi OE, Camici PG, et al. Functional changes in coronary microcirculation after valve replacement in patients with aortic stenosis. Circulation 2003;107:3170-5.

8. Di Gioia G, Pellicano M, Toth GG, et al. Fractional Flow Reserve-Guided Revascularization in Patients With Aortic Stenosis. Am J Cardiol 2016;117:1511-5.

9. Dewey TM, Brown DL, Herbert MA, et al. Effect of concomitant coronary artery disease on procedural and late outcomes of transcatheter aortic valve implantation. Ann Thorac Surg 2010;89:758-67; discussion 767.

10. Masson JB, Lee M, Boone RH, et al. Impact of coronary artery disease on outcomes after transcatheter aortic valve implantation. Catheter Cardiovasc Interv 2010;76:165-73.

11. Wenaweser P, Pilgrim T, Guerios E, et al. Impact of coronary artery disease and percutaneous coronary intervention on outcomes in patients with severe aortic stenosis undergoing transcatheter aortic valve implantation. EuroIntervention 2011;7:541-8.

12. Abdel-Wahab M, Mostafa AE, Geist V, et al. Comparison of outcomes in patients having isolated transcatheter aortic valve implantation versus combined with preprocedural percutaneous coronary intervention. Am J Cardiol 2012;109:581-6.

13. Gautier M, Pepin M, Himbert D, et al. Impact of coronary artery disease on indications for transcatheter aortic valve implantation and on procedural outcomes. EuroIntervention 2011;7:549-55.

14. Goel SS, Agarwal S, Tuzcu EM, et al. Percutaneous coronary intervention in patients with severe aortic stenosis: implications for transcatheter aortic valve replacement. Circulation 2012;125:1005-13.

15. Paradis JM, White JM, Genereux P, et al. Impact of Coronary Artery Disease Severity Assessed With the SYNTAX Score on Outcomes Following Transcatheter Aortic Valve Replacement. J Am Heart Assoc 2017;6.

16. Witberg G, Lavi I, Harari E, et al. Effect of coronary artery disease severity and revascularization completeness on 2-year clinical outcomes in patients undergoing transcatether aortic valve replacement. Coron Artery Dis 2015;26:573-82.

17. Witberg G, Regev E, Chen S, et al. The Prognostic Effects of Coronary Disease Severity and Completeness of Revascularization on Mortality in Patients Undergoing Transcatheter Aortic Valve Replacement. JACC Cardiovasc Interv 2017;10:1428-35.

18. Chakravarty T, Sharma R, Abramowitz Y, et al. Outcomes in Patients With Transcatheter Aortic Valve Replacement and Left Main Stenting: The TAVR-LM Registry. J Am Coll Cardiol 2016;67:951-60.

19. Conradi L, Seiffert M, Franzen O, et al. First experience with transcatheter aortic valve implantation and concomitant percutaneous coronary intervention. Clin Res Cardiol 2011;100:311-6.

20. Pasic M, Dreysse S, Unbehaun A, et al. Combined elective percutaneous coronary intervention and transapical transcatheter aortic valve implantation. Interact Cardiovasc Thorac Surg 2012;14:463-8.

21. Singh V, Rodriguez AP, Thakkar B, et al. Comparison of Outcomes of Transcatheter Aortic Valve Replacement Plus Percutaneous Coronary Intervention Versus Transcatheter Aortic Valve Replacement Alone in the United States. Am J Cardiol 2016;118:1698-704.

22. Kotronias RA, Kwok CS, George S, et al. Transcatheter Aortic Valve Implantation With or Without Percutaneous Coronary Artery Revascularization Strategy: A Systematic Review and Meta-Analysis. J Am Heart Assoc 2017;6. doi: 10.1161/JAHA.117.005960.

23. Yudi MB, Sharma SK, Tang GHL, et al. Coronary Angiography and Percutaneous Coronary Intervention After Transcatheter Aortic Valve Replacement. J Am Coll Cardiol 2018;71:1360-78.

24. Palmerini T, Biondi-Zoccai G, Della Riva D, et al. Stent 
thrombosis with drug-eluting stents: is the paradigm shifting? J Am Coll Cardiol 2013;62:1915-21.

25. Abramowitz Y, Chakravarty T, Jilaihawi H, et al. Clinical impact of coronary protection during transcatheter aortic valve implantation: first reported series of patients. EuroIntervention 2015;11:572-81.

26. Harhash A, Ansari J, Mandel L, et al. STEMI After TAVR: Procedural Challenge and Catastrophic Outcome. JACC Cardiovasc Interv 2016;9:1412-3.

27. Khawaja MZ, Wang D, Pocock S, et al. The percutaneous

Cite this article as: Perez S, Thielhelm TP, Cohen MG. To revascularize or not before transcatheter aortic valve implantation? J Thorac Dis 2018;10(Suppl 30):S3578-S3587. doi: 10.21037/jtd.2018.09.85 coronary intervention prior to transcatheter aortic valve implantation (ACTIVATION) trial: study protocol for a randomized controlled trial. Trials 2014;15:300.

28. Ramee S, Anwaruddin S, Kumar G, et al. The Rationale for Performance of Coronary Angiography and Stenting Before Transcatheter Aortic Valve Replacement: From the Interventional Section Leadership Council of the American College of Cardiology. JACC Cardiovasc Interv 2016;9:2371-5. 